\title{
KALMAN FILTER IMPLEMENTATION FOR UNMANNED AERIAL VEHICLES NAVIGATION DEVELOPED WITHIN A GRADUATE COURSE
}

\author{
Vasko Sazdovski, Tatjana Kolemishevska-Gugulovska, Mile Stankovski \\ Institute of ASE at Faculty of EE,, St. Cyril and Methodius University, MK-1000, Skopje, Republic of \\ Macedonia; E-mail:[milestk, tanjakg]@etf.ukim.edu.mk
}

\begin{abstract}
Part-time studies of the MSc program as source of number of technical and scientific innovations in Automation and Computer Based Systems Engineering at Faculty of Electrical Engineering of SS Cyril and Methodius University in Skopje, are run for more than 15 years. This innovation in Unmanned Aerial Vehicles (UAV's) is the most recent one. By definition, navigation is a process of determining the navigational parameters of the centre of mass, of the moving object. The system that provides us the navigational parameters is called Navigation System. The most used Navigation Systems, developed for a wide range of vehicles, are Inertial Navigation System (INS) and Global Positioning System (GPS). These two systems have their advantages and disadvantages, which makes them complementary. The best estimates of the aircraft position, velocity and attitude can be obtained by GPS/INS integration employing Kalman filter implementation. Kalman filtering is a form of optimal estimation characterized by recursive evaluation, and an internal model of the dynamics of the system being estimated Copyright $(2005$ IFAC.
\end{abstract}

Keywords: Kalman filters, inertial navigation, Global positioning systems, aerospace control, simulation

\section{INTRODUCTION}

Unmanned Aerial Vehicles (UAV), such as spacecrafts, aircrafts, helicopters, free-flying robots or mobile robots are increasingly applied in various domains, particularly in the military, scientific research, and in certain industries. By definition, navigation is a process of determining the current parameters of movement, like accelerations, velocity and position of center of mass, of the moving object. The system that provides us with navigational parameters is called Navigation System. One of the most used Navigation Systems, which is developed for a wide range of vehicles, is the Inertial Navigation System (INS). Other system, which is the most famous, is the Global Positioning System (GPS). This two systems have their advantages and disadvantages, which makes them complementary, and the best estimates of the aircraft position, velocity and attitude can be obtained by GPS/INS integration, with Kalman filter implementation. The purpose of combining navigation subsystems into an integrated system is to take advantage of complementary strengths of the subsystems.
Kalman filtering is a form of optimal estimation characterized by recursive evaluation, and an internal model of the dynamics of the system being estimated. Kalman filter has the ability to combine the subsystems, on the knowledge of the measurements noise covariance (GPS measurements noise covariance) and the process noise covariance.

\section{NAVIGATION SYSTEMS}

\subsection{Inertial Navigation System (INS)}

The INS algorithm integrates the accelerations and angular rates provided by an Inertial Measurement Unit (IMU) to compute the position, velocity, and attitude (PVA) of the vehicle (Lin 1991).

The algorithm takes into account the geoid shape and a gravity model. A basic flow chart of the operation of an inertial navigation system is shown on Fig. I. There are many problems with noise and unbounded error that must be handled to get any meaningful result out of the INS. 


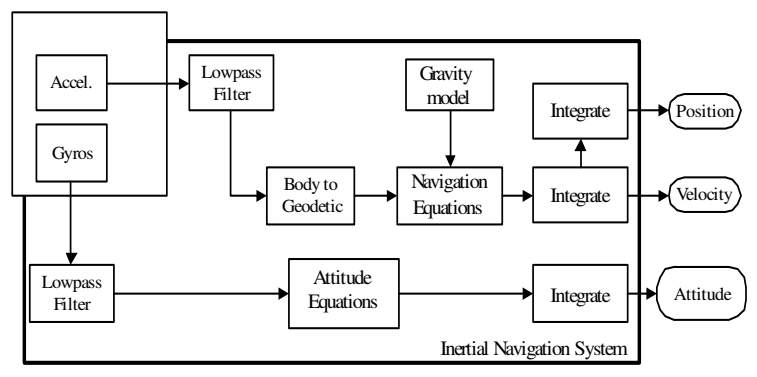

Fig.I. A flow chart of a strap-down INS

Reference Frames and Rotations. For the purposes of this work, several reference frames were used, see figure II. Earth Centered Earth Fixed Frame $E\left(O_{e} ; x_{e} ; y_{e} ; z_{e}\right)$, Normal Earth Fixed Axis System $G_{o}\left(0 ; x_{0} ; y_{0} ; z_{0}\right)$ then Aircraft Carried Normal Earth Axis System $G\left(P ; x_{g} ; y_{g} ; z_{g}\right)$ also known as Geodetic frame and Body frame $B(P ; x ; y ; z)$. All the frames are in accordance with the ISO standards.

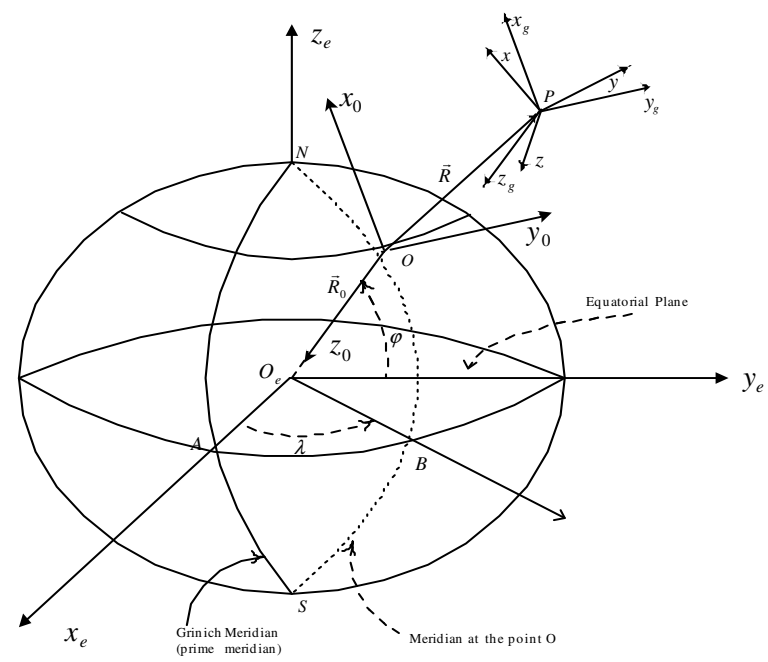

Fig. II. Reference frames used in INS

As a result from the transition between the various reference frames several rotation matrices were developed (Deskovski 2004). The first one takes measurements in the body frame and puts them into the Geodetic frame.

$T_{G B}=\left[\begin{array}{ccc}\cos \theta \cos \psi & \sin \phi \sin \theta \cos \psi-\cos \phi \sin \psi & \sin \phi \sin \psi+\cos \phi \sin \theta \cos \psi \\ \cos \theta \sin \psi & \cos \phi \cos \psi+\sin \phi \sin \theta \sin \psi & \cos \phi \sin \theta \sin \psi-\sin \phi \cos \psi \\ -\sin \theta & \sin \phi \cos \theta & \cos \phi \cos \theta\end{array}\right]$

where $\phi$ is a roll, $\theta$ is a pitch, and $\psi$ is a yaw. This rotation is the sequence 1-2-3, which is typically used in aerospace applications. The next rotation will transform points from the ECEF frame to the Geodetic frame.

$$
T_{G E}=\left[\begin{array}{rrrr}
-\sin \phi \cos \lambda & -\sin \phi \sin \lambda & \cos \phi \\
-\sin \lambda & \cos \lambda & 0 \\
-\cos \phi \cos \lambda & -\cos \phi \sin \lambda & -\sin \phi
\end{array}\right]
$$

where $\varphi$ is a latitude and $\lambda$ is a longitude.

Navigation Equations. The information provided by the IMU such as body accelerations are transformed to navigation frame and gravity vector is subtracted. The resulting acceleration vector is integrated with respect to time and we get the velocity of the vehicle. The velocity vector is then integrated and we can read the position of the vehicle. Navigation equations used in this work are:

$\left[\begin{array}{c}\dot{x}_{0} \\ \dot{y}_{0} \\ \dot{z}_{0} \\ \dot{V}_{x g} \\ \dot{V}_{y g} \\ \dot{V}_{z g}\end{array}\right]=\left[\begin{array}{cccccc}0 & 0 & 0 & 1 & 0 & 0 \\ 0 & 0 & 0 & 0 & 1 & 0 \\ 0 & 0 & 0 & 0 & 0 & 1 \\ 0 & 0 & 0 & 0 & r_{g} & -q_{g} \\ 0 & 0 & 0 & -r_{g} & 0 & p_{g} \\ 0 & 0 & 0 & q_{g} & -p_{g} & 0\end{array}\right] \cdot\left[\begin{array}{c}x_{0} \\ y_{0} \\ z_{0} \\ V_{x g} \\ V_{y g} \\ V_{z g}\end{array}\right]+$

$\left[\begin{array}{cccccc}0 & 0 & 0 & 0 & 0 & 0 \\ 0 & 0 & 0 & 0 & 0 & 0 \\ 0 & 0 & 0 & 0 & 0 & 0 \\ \left(q_{0}^{2}+q_{1}^{2}-q_{2}^{2}-q_{3}^{2}\right) & 2\left(q_{1} q_{2}-q_{0} q_{3}\right) & 2\left(q_{1} q_{3}+q_{0} q_{2}\right) & 1 & 0 & 0 \\ 2\left(q_{1} q_{2}+q_{0} q_{3}\right) & q_{0}^{2}-q_{1}^{2}+q_{2}^{2}-q_{3}^{2} & 2\left(q_{2} q_{3}-q_{0} q_{1}\right) & 0 & 1 & 0 \\ 2\left(q_{1} q_{3}-q_{0} q_{2}\right) & 2\left(q_{2} q_{3}+q_{0} q_{1}\right) & \left(q_{0}^{2}-q_{1}^{2}-q_{2}^{2}+q_{3}^{2}\right) & 0 & 0 & 1\end{array}\right] \cdot\left[\begin{array}{c}a_{x} \\ a_{y} \\ a_{z} \\ 0 \\ 0 \\ -g\end{array}\right]$

where $p_{g}, q_{g}$ and $r_{g}$ are rotation rates in Geodetic frame. If the navigation system is ment to work over longer distances we need to take in calculation the Corriolis acceleration caused by the rotation of the Earth. In this work Corriolis effect was not considered.

Sources of Error. There are many difficulties which are present in the INS. Accelerometer bias, then gyros drift, temperature and vibrations. In this work because of simplicity only the accelerometer bias is included.

The accelerometer bias has a quadratic effect on the position derived from the IMU (Walchko, 2002),

$$
\text { error }=\frac{1}{2} \text { bias } \cdot t^{2}
$$

\begin{tabular}{lcc}
\hline $\begin{array}{l}\text { Bias } \\
\mathrm{m} / \mathrm{s}^{2}\end{array}$ & $\begin{array}{c}\text { Error }(\mathrm{m}) \\
\mathrm{t}=100 \mathrm{sec}\end{array}$ & $\begin{array}{c}\text { Error }(\mathrm{m}) \\
\mathrm{t}=30 \mathrm{~min}\end{array}$ \\
\hline 0.1 & 500 & 162000 \\
0.01 & 5 & 16200 \\
0.001 & 0.5 & 1620 \\
0.0001 & 0.05 & 162 \\
\hline
\end{tabular}

Table 1. Positional error that results from biases after a time of 100 seconds and $30 \mathrm{~min}$

Looking at the table 1 it becomes apparent that the determination of the bias is of critical importance if any accurate measurement is expected. 


\subsection{Global Positioning System (GPS)}

Global Positioning System (GPS) can be regarded as a new navigation sensor. GPS provides range and range-rate measurements. The primary role of GPS is to provide highly accurate position and velocity worldwide, based on range and range-rate measurements. The acceleration vector is then determined from positions at different time epochs, by differentiation of these positions with respect to time. Position accuracy of GPS pseudo-range absolute positioning is affected by measurement noise (few metres) and signal errors like: multipath of the signal, ionosphere delays, troposphere delays, signal attenuation, ephemeris error, satellite clock error and receiver clock error (Ronback, 2002). Also, the GPS signal is susceptible to jamming. For many vehicle navigation systems, GPS is insufficient as a stand alone position system.

\section{KALMAN FILTER}

The Kalman filter (Gene et al. 2000) assumes that the random process which has to be estimated is of the form:

$$
\dot{x}=F x+B u+G w
$$

where $x$ is a state value, $u$ is a control effort, $w$ is white noise with known covariance.

When measurements are taken of the process at discrete moments in time, they occur according to the following relationship:

$$
z=H x+D u+v
$$

where $\mathrm{z}$ is a noisy sample, $D$ is the direct transmission of the input to the output, $H$ is the ideal (noiseless) connection between the measurement and the state, and $v$ is measurement error.

This process can be modeled discretely in the following form, assuming there are not control inputs $u$ to the system.

$$
\begin{aligned}
& x_{K+1}=\Phi_{K} x_{K}+w_{K} \\
& z_{K}=H_{K} x_{K}+v_{K}
\end{aligned}
$$

The system error is defined as:

$$
e_{K}^{-}=x_{K}-\hat{x}_{K}^{-}
$$

where $\hat{x}_{k}^{-}$is the best estimate prior to receiving a measurement at time $t_{K}$.

The error covariance matrix at this time is:

$$
P_{K}^{-}=E\left[e_{K}^{-} e_{K}^{-T}\right]=E\left[\left(x_{K}-\hat{x}_{K}^{-}\right)\left(x_{K}-\hat{x}_{K}^{-}\right)^{T}\right]
$$

where $E$ is the expectation.
Now a linear blending of both the estimate and the measured value is taken.

$$
\hat{x}_{K}=\hat{x}_{K}^{-}+K_{K}\left(z_{K}-H_{K} \hat{x}_{K}^{-}\right)
$$

where $\hat{x}_{K}$ is the new updated estimate, $z$ is measured value, and $K$ is a weighted value that determines the amount of error between the measured value and the best estimate.

This gain is referred to as the Kalman gain which is capable of changing value over time.

Now looking at the error covariance of this new updated estimate, we get the following equation:

$$
P_{K}=E\left[e_{K} e_{K}^{T}\right]=E\left[\left(x_{K}-\hat{x}_{K}\right)\left(x_{K}-\hat{x}_{K}\right)^{T}\right]
$$

Now, after some algebra the following expression results for the error covariance matrix:

$$
P_{K}=\left(I-K_{K} H_{K}\right) P_{K}^{-}\left(I-K_{K} H_{K}\right)^{T}+K_{K} R_{K} K_{K}
$$

This is a general expression for updating the error covariance matrix, and it applies for any value of $K$. The resulting gain $\mathrm{K}$ is computed by the equation:

$$
K_{K}=P_{K}^{-} H_{K}^{-}\left(H_{K} P_{K}^{-}-H_{K}^{T}+R_{K}\right)^{-1} \text {. }
$$

\section{SIMULATION MODELS OF INTEGRATED GPS/INS NAVIGATION SYSTEM}

When we make the simulation models we use the modular approach, where all systems are running independent. Modular approach let us to enhancement the models easy and also to substitute easy the systems in the current models.

Simulation model of an Integrated GPS/INS Navigation System is shown on figure II. Simulation model is realized in Matlab-Simulink and we can see that the model includes Navigation Linear Error Kalman Filter.

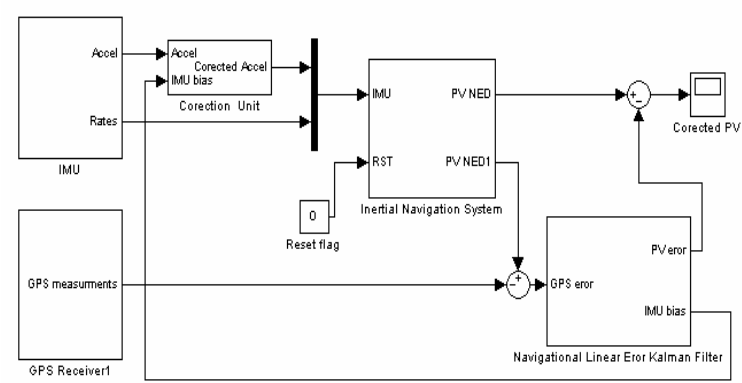

Fig. III Simulation model of Integrated GPS/INS Navigation System

Equations used in the Kalman filter are:

$$
\left[\begin{array}{c}
\Delta \dot{R} \\
\Delta \dot{V} \\
\Delta \dot{b}
\end{array}\right]=\left[\begin{array}{lll}
0 & 1 & 0 \\
0 & 0 & 1 \\
0 & 0 & 0
\end{array}\right]\left[\begin{array}{c}
\Delta R \\
\Delta V \\
\Delta b
\end{array}\right]+\left[\begin{array}{c}
0 \\
n_{r} \\
n_{w}
\end{array}\right],
$$


and measurement equation:

$$
\Delta z=\left[\begin{array}{lll}
1 & 0 & 0 \\
0 & 1 & 0
\end{array}\right]\left[\begin{array}{c}
\Delta R \\
\Delta V \\
\Delta b
\end{array}\right]+n_{v}
$$

where $\Delta R$ is the position error, $\Delta V$ is the velocity error and $\Delta b$ is accelerometer bias, and $n_{r}, n_{w}, n_{v}$ are Gaussian white noises, respectively, with known covariances (Roumeliotis et. Al 1998).

From fig. III we can see that estimated errors in position and velocity are subtracted from the position and velocity of the INS. Also, estimated bias in the correction unit is subtracted from the outputs from the IMU. The disadvantage of the Navigation Linear Error Kalman Filter is that the assumption of linearity for the error estimates is not always valid. Also, the unbounded error in the uncorrected INS output can cause numerical problems if the algorithm will run for a long time.

Simulation model of an Integrated GPS/INS Navigation System with included Navigation Extended Kalman Filter is shown on figure IV. Simulation model is realized in Matlab-Simulink. Navigation Extended Kalman Filter differs from the Navigation Linear Error Kalman Filter.

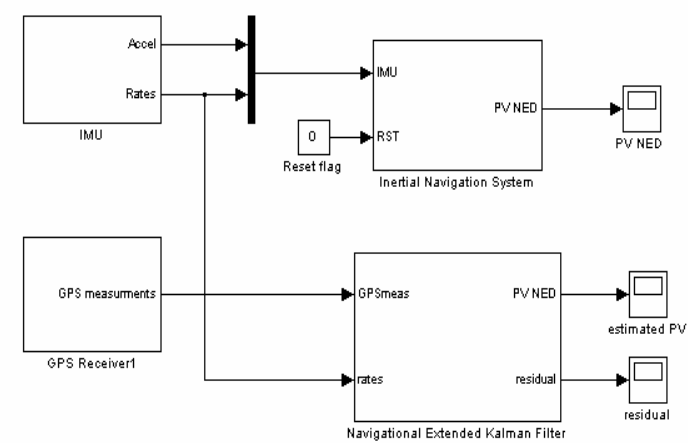

Fig. IV Simulation model of Integrated GPS/INS Navigation System

The Information's from the GPS receiver (position and velocity) are observations to the Kalman filter. Extended Kalman filter is realized as an M-file and it is executed as a Matlab function block in the simulation model, see figure $\mathrm{V}$.

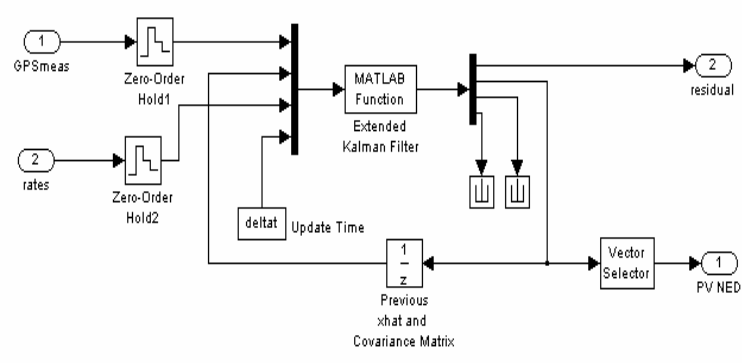

Fig. V. Extended Kalman filter realized as a Matlab M-file and executed as a Matlab function block
The advantage of the nonlinear Kalman filters is that they can directly estimate the vehicle dynamics (which are non-linear in most cases). Both the vehicle states and the sensor measurement equations can have nonlinear terms. This results in better estimation accuracy, over a wider range of operating conditions. The main disadvantage of the nonlinear Kalman filters is that the algorithms are more complex than the linear implementation, therefore requiring more computational resources.

\section{SIMULATION EXPERIMENTS AND RESULTS}

Two simulation models were tested, and two cases were considered. In the first case the difference between IMU and GPS measurements is constant and it is 10 meters. The second case is different and the difference between INS and GPS change in the time and in some moments of time reaches 50 meters.

In the simulation UAV is climbing with acceleration $a_{z}=3 g$. The bias in the Linear Error Kalman filter is taken that has covariance $0.0049 \mathrm{~m} / \mathrm{s}^{2}$ (Vanicek and Omerbasic, 2002).

The covariance of the noise in the IMU is taken that is $Q=0.0012 \mathrm{~m} / \mathrm{s}^{2}$ (process noise), and the covariance of the noise in the GPS receiver is $R=5 m$. (measurement noise).

Results of the simulation of the Navigational Linear Error Kalman filter are shown. Figure VI and VII show the true trajectory and estimated trajectory of the vehicle in the first case and in the second case, respectively. The two smooth curves are the true trajectory and the estimated trajectory, and they are almost too close to distinguish from one another.

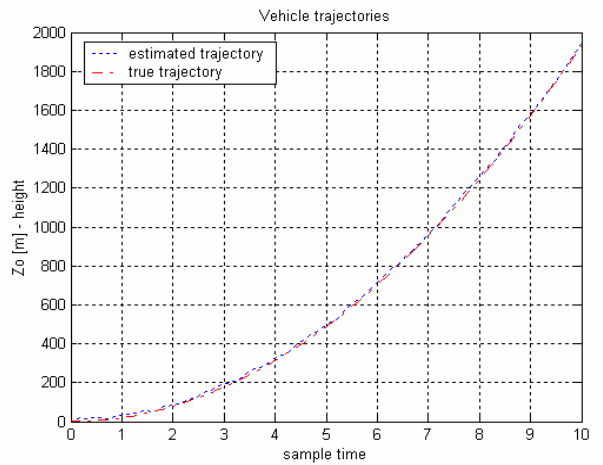

Fig. VI. Trajectories of the vehicle in the first case

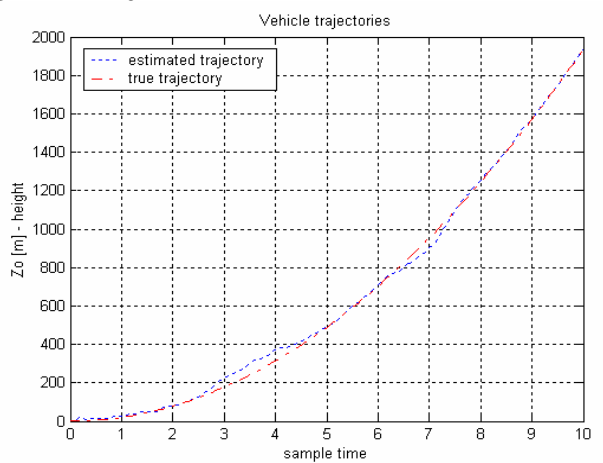

Fig. VII Trajectories of the vehicle in the second case 


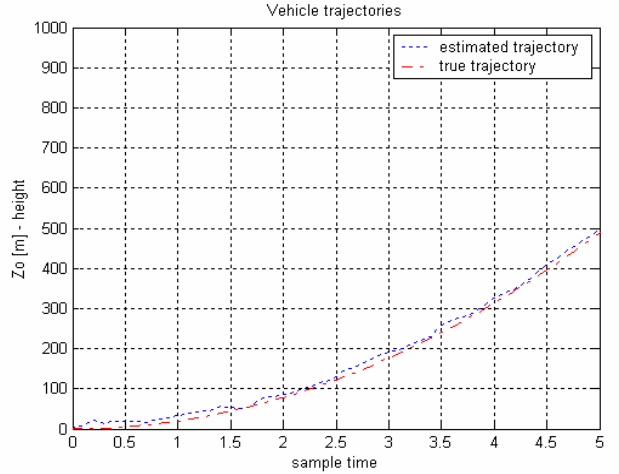

Fig. VIII Closer look of the trajectories in the first case

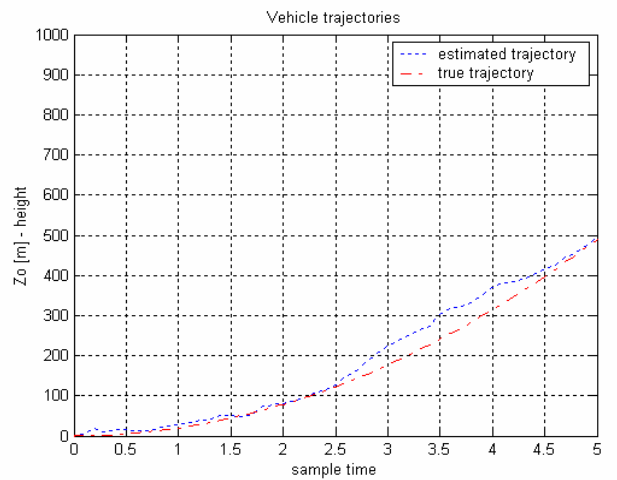

Fig. IX Closer look of the trajectories in the second case

The diffrence between the true and estimated trajectories is better visible on figures VIII and IX which present closer look of the vehicle trajectories.

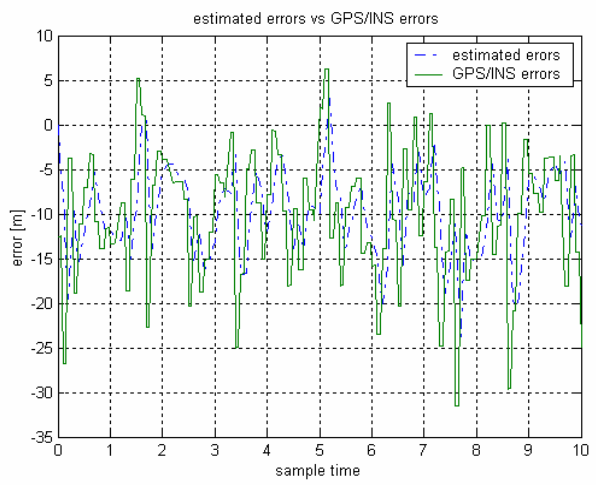

Fig. X GPS/INS errors vs. estimated errors in the first case

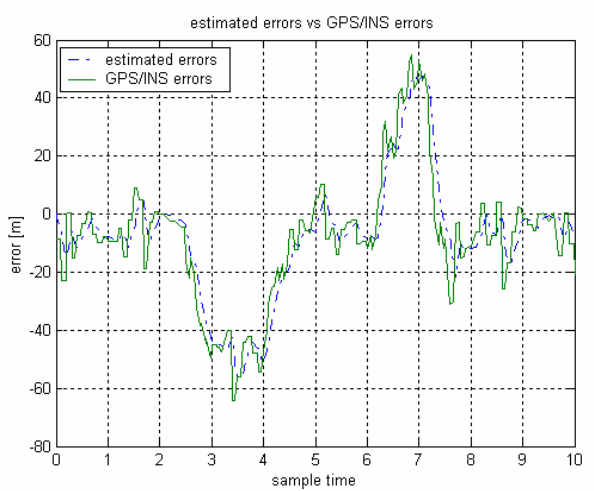

Fig. XI GPS/INS errors vs. estimated errors in the second case
The GPS/INS errors vs. estimated errors, in the two cases are shown on Fig. X and XI.

In the beginning of the simulation we see that estimated errors are zero, so we predict perfect estimation. The GPS/INS errors and estimated errors have a standard deviation of about 10 meters in the first case and in the second case, errors have occasional spikes up to 50 meters.

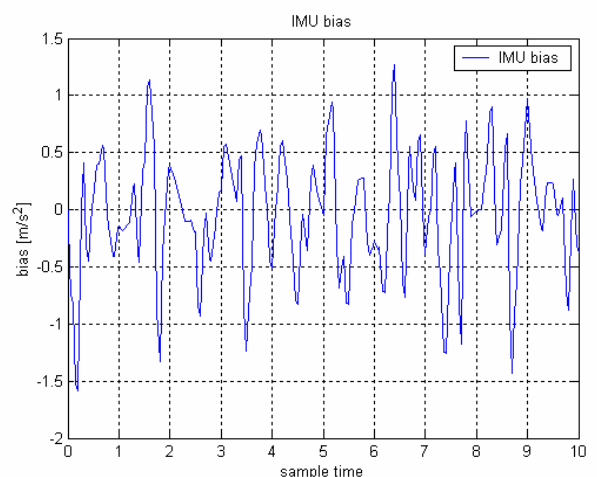

Fig.XII. Changes of IMU bias in the first case

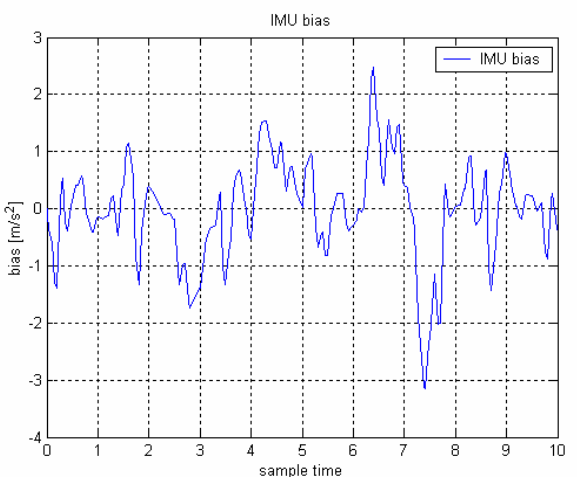

Fig. XIII. Changes of IMU bias in the second case

The changes of IMU bias are presented on fig. XII and XIII for the two cases. From where we can see the importance of augmenting the system model with the bias model. Estimated bias is subtracted from the outputs from the IMU to reduce errors.

The true and estimated trajectories of the UAV when the Extended Kalman filter was simulated are shown on figure XIV and XV. The two smooth curves are the true trajectory and the estimated trajectory, and they are almost too close to distinguish from one another.

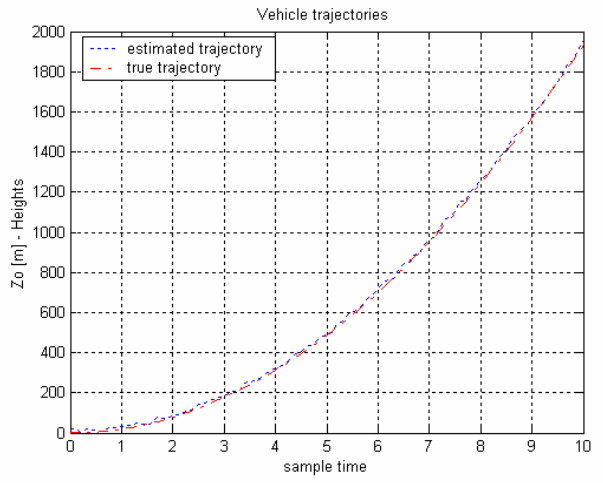

Fig. XIV Trajectories of the vehicle in the first case 


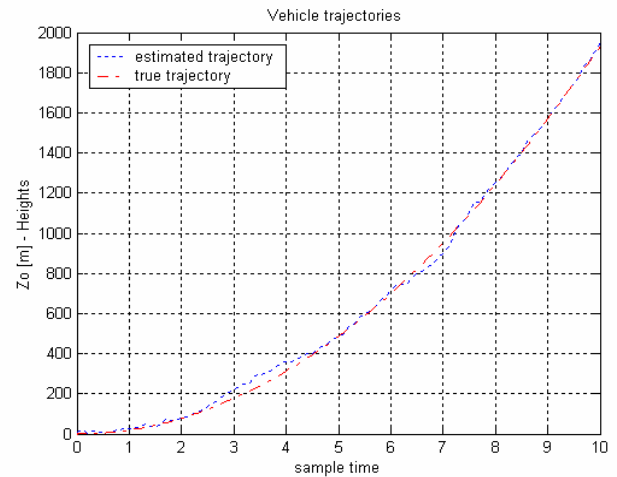

Fig. XV Trajectories of the vehicle in the second case

The difference between the true and estimated trajectories is better visible on figures XVI and XVII which present closer look of the vehicle trajectories.

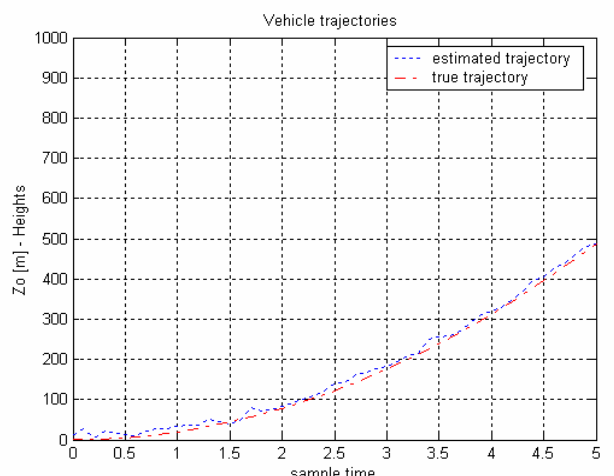

Fig. XVI Closer look of the trajectories in the first case

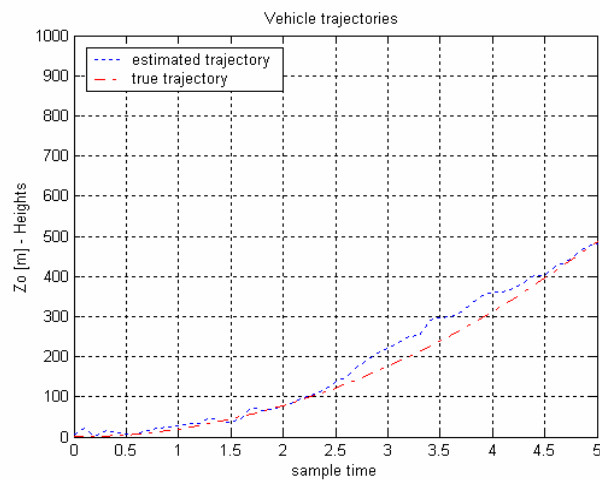

Fig. XVII Closer look of the trajectories in the second case

On figures XVIII and XIX changes of the residual in the Extended Kalman filter, in the two cases are shown.

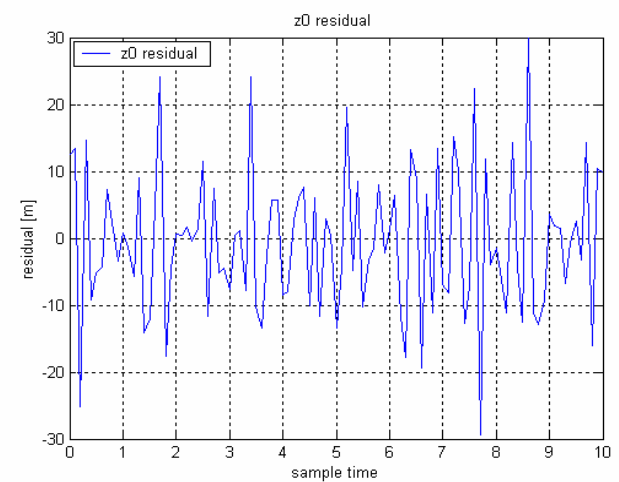

Fig. XVIII Changes of the residual in the first case

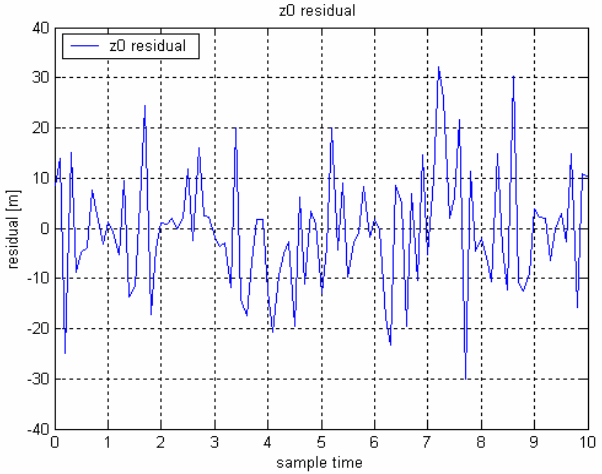

Fig. XIX. Changes of the residual in the second case

\section{CONCLUSIONS AND FUTURE WORK}

In this work two simulation models of Integrated Navigational Systems were presented. Program realization was in Matlab-Simulink, were the two Kalman filter algorithms were tested. Simulation results show the Kalman filter efficiency and therefore the efficiency of the Integrated Navigation Systems.

The aim of the simulation experiments was getting optimal results, as first phase of development of the Integrated Navigation Systems.

Future work will include identifying a low-power microprocessor capable of running the Kalman Filters in real-time at desired rate and testing in hardware-in-the-loop simulation.

Simulation models will be enhanced by adding others systems (sensors), also Kalman filters will be enhanced with fuzzy logic.

\section{REFERENCES}

Gene F. Franklin., J.David Powell., Michael L. Workman.: (2000) Digital Control of Dynamic Systems, Second edition, Addison-Wesley.

Lin, C. F.: (1991), Modern Navigation, Guidance, and Control Procesing, Volume II, Prentice Hall, Englewood Cliffs.

Petr Vanicek, Mensur Omerbasic, (2002) Does a navigation algorithm have to use Kalman filter, Department of Geodesy and Geometics Engineering, University of New Brunswick, Canada.

Deskovski S., (2004) Homing guidance and Air Defence Systems, Bata press, Skopje.

Walchko K. J., (2002), Low cost inertial navigation: Lerning to integrate noise and find your way, Univeresity of Florida.

Ronback., S., (2002), Develpoment of a INS/GPS navigation loop for an UAV, master thesis, Lulea University of technology, Hungary.

Roumeliotis, S. I., Gaurav S. Sukhatme, George A. Bekey., (1998), Circumventing Dynamic Modeling: Evaluation of the eror-state Kalman filter applied to Mobile Robot Localization, University of Southern California 